\title{
Differential common path interferometry for picometre surface metrology
}

A. Schultze, A. Sell, H. Kögel, D. Weise, C. Braxmaier

A. Schultze, A. Sell, H. Kögel, D. Weise, C. Braxmaier, "Differential common path interferometry for picometre surface metrology," Proc. SPIE 11852, International Conference on Space Optics - ICSO 2020, 118526N (11 June 2021); doi: 10.1117/12.2600308

SPIE Event: International Conference on Space Optics - ICSO 2021, 2021, Online Only 


\section{International Conference on Space Optics-ICSO 2020}

Virtual Conference

30 March-2 April 2021

Edited by Bruno Cugny, Zoran Sodnik, and Nikos Karafolas
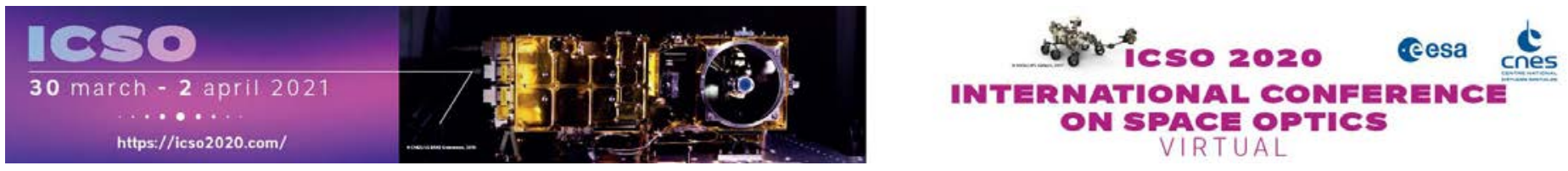

\section{Differential common path interferometry for picometre surface metrology}

\section{Cesa isoporecestings denes}




\title{
Differential common path interferometry for picometre surface metrology
}

\author{
A. Schultze ${ }^{\text {a }}$ A. Sell ${ }^{\mathrm{a}}$, H. Kögel ${ }^{\mathrm{a}}$, D. Weise ${ }^{\mathrm{a}}$, and C. Braxmaier ${ }^{\mathrm{b}, \mathrm{c}}$

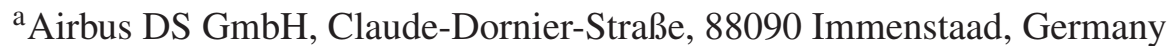 \\ ${ }^{\mathrm{b} U n i v e r s i t a ̈ t ~ B r e m e n, ~ Z A R M, ~ A m ~ F a l l t u r m ~ 2, ~} 28359$ Bremen, Germany \\ ${ }^{\mathrm{c} D L R}$ Institut für Raumfahrtsysteme, Robert-Hooke-Str. 7, 28359 Bremen, Germany \\ *Contact: alexander.schultze@ airbus.com
}

\begin{abstract}
Future space based missions for gravitational wave research call for an improved inertial reference sensor with acceleration noise levels of $\mathrm{fm} / \mathrm{s}^{2}$. Spherical test masses can enable increased performance by suspension-free operation, contrary to cuboid solutions suffering from cross-coupling of attitude control noise. However, interferometric readout is affected by surface irregularities and test mass tumbling [1]. An accurate surface map for compensation must be established for compensation, either by characterisation a priori or in flight, when optical path length changes due to surface occur in the measurement band.

We demonstrate a method for generating a surface map of a spherical body with optical point sensors using a differential method to suppress common mode errors present, taking advantage of the excellent performance of heterodyne interferometry at sub-nanometer levels. A measurement setup is proposed in which two beams of a Nd:YAG Michelson interferometer are used to scan the surface, which is afterwards reconstructed from the differential measurement.

Such a method could potentially benefit other research areas, such as the precise determinations of the Avogadro constant [2] or aspheric surface metrology.
\end{abstract}

Keywords: Spherical Test Mass, Surface Metrology, Heterodyne Interferometry, Gravitational Reference Sensor, Surface Map

\section{INTRODUCTION}

On-ground surface calibration with an optical point scanning approach is challenging due to the need for mechanical movement, but can profit from the high accuracy of heterodyne optical interferometers. The demonstrated performance [3] at pm levels by using separate-path heterodyne interferometry makes optical point metrology an interesting candidate to enable precise surface topography mapping.

The basic principle proposed is shown in fig. 1. It employs two differential laser beams (red), with one additional beam (green) for compensation of cross-axes coupling. The two beams of the differential leg pass through a focusing lens and are reflected on the surface. The so measured surface difference is used to reconstruct the surface profile of a circle of latitude. This technique implements a common mode rejection of error movements in measurement direction as well as long-term drifts incident in a single path setup featuring a reference mirror. Currently the performance is limited by the auxiliary lateral measurement, leading to an improvement of a factor of $\sim 10$ in the differential data. The measurement precision of the differential measurement is improved compared to previous absolute measurements with repeatability at nanometer levels. By combining several circle measurement at different elevations, a full circle surface profile can be reconstructed. Precise surface maps are a prerequisite

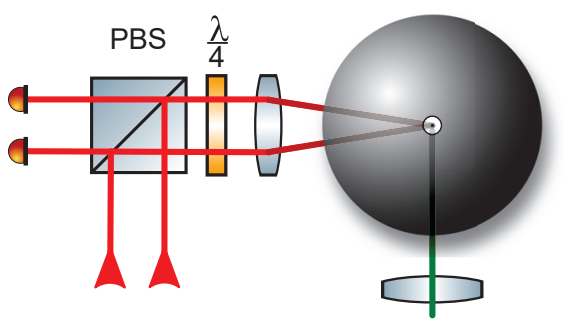

Figure 1: Schematic measurement setup.

step to enable slowly rotating spherical test masses for future gravitational research missions. A Gravitional Reference Sensor (GRS), composed of a test mass and its read out, is an elementary part of the Drag-free Attitude 
Control System (DFACS) for space borne gravitational wave and gravity research missions. The test masses are floating force-free in space and define the reference point for the scientific acceleration measurements that allow to detect gravitational waves. The required sensitivity of the translation readout of these test masses is $\mathrm{pm} / \sqrt{\mathrm{Hz}}$. For the Laser Interferometer Space Antenna (LISA) mission the baseline are two cuboid test masses of a gold-platin alloy, one for each interferometer arm, that during science mode need to be jointly controlled in rotational and translational degrees of freedom (except only for lines of sight). It has been proposed to use spherical test masses, in which case a single test mass can be used as an endpoint, with no need for active control of any degree of freedom [1]. The optical readout for the free floating test mass of the GRS would benefit from the reduced acceleration noise. An alternative that does not require prior mapping if the surface is to spin the sphere fast enough to shift surface effects above the measurement band [4].

Test mass shape and surface topology introduce path length errors caused by a freely moving test mass, which are suppressed in a concept featuring an attitude controlled proof mass, as well as residual tilt-to-length effects in the interferometer optics.

The influence of sphere rotation and translation on the optical path-length and beam deflection need to be understood, both for the determination of the map as well as the readout of a future GRS.

Current state-of-the-art surface metrology can be categorised into tactile, optical point and optical area sensors. Optical area sensors can be further divided into methods using condensing lens, sub-aperture section (SSI) or more recently introduced Computer Generated Hologram (CGH) based techniques with diffractive lenses. These methods achieve performance levels down to down to $5 . .91 \mathrm{~nm}$ (rms) or 51..1095 $\mathrm{nm}$ peak valley [5].

Some recent publications focus on measurement of diameter topographies instead of surface topographies [6] [7]. However, the functional principle of a GRS requires a surface map representing the actual surface topography to compensate for path length effects.

Another differential picometer profiliometry approach is described in [8], where the use of a Wollaston prism allows beams to scan in very close proximity due to the polarization routing.

\section{METHODOLOGY}

The surface measurement proposed covers the whole sphere by combining rings recorded via high accuracy differential circle profilometry. For each ring, the sphere is rotated around the azimuth axis by a rotation stage mechanism. A differential measurement monitoring two points offset laterally can be used to reconstruct a surface profile, either using a discrete reconstruction approach or operating in Fourier space. Several great circles at different elevation positions are then combined into a sphere surface using a stitching combination. This method benefits from a high rejection of common path length errors in the differential part of the setup as well as very low noise levels.

The orientation of the measurement laser beams is shown in figure 3. Two parallel beams are oriented at the same height with a azimuthal separation. The lens performs wavefront matching of the beam and geometrical centering towards the center of the sphere. The two beams probe the optical surface on two adjacents surface spots. With a complete measurements of surface profile differences around one circle, the surface can be recovered using integration methods.

The circle is measured by rotating the sphere under test with a constant velocity on a precision rotary stage. The measured ring is ideally a great circle of the sphere. The measurement is naturally independent of common path errors colinear to the parallel beams direction, but non common path effects can still be introduced by transversal movement. Simplified, the path length is impacted with a factor of $(1-\cos (y))$, where $y$ is the sphere sideways motion in the measurement plane. Additional effects must be considered for varying surface normals causing tilt to length effects.

To correct for this additional path length, a second interferometer perpendicular to the first and the rotation axis is installed, monitoring the movement along $\mathrm{y}$ in the measurement plane. By acquiring several great circles while slightly rotating the sphere around a second axis orthogonal to the first rotation axis, the surface can be stitched together from multiple reconstructed rings.

\section{SETUP}

Heterodyne interferometer The interferometer setups used are two heterodyne symmetrical Michelsen interferometers with spacially seperated beams. A detailed description of the interferometer is given in [9]. The setup is shown in figure 3 and identical for both interferometers \#1 and \#2. The setup uses stabilised laser light from a non-planar ring-oscillator Nd:YAG laser (1064 nm), which is actively frequency-locked onto an optical cavity by Pound-DreverHall (PDH) technique. The heterodyne frequencies are generated by frequency-shifting via acousto optic modulators (AOMs) with frequencies of $f_{\text {aom } 1 / 2}=78.00 / 78.01 \mathrm{MHz}$ generating a beat at $10 \mathrm{kHz}$. The photodiode channels are read out at a rate of $160 \mathrm{kHz}$ and IQ demodulated on a Field Programmable Gate Array (FPGA) with a $10 \mathrm{kHz}$ reference signal. The electrical readout chain and the heterodyne frequency generators are synchronised to a Rubidium frequency standard. 
Each interferometer has 3 readout ports, one local readout and two measurement readouts. The local path length measurement is used for phase locking the fibre output, and as a reference for the optical path lengths for measurement.

Performance for such interferometers has been demonstrated to noise levels of $<1 \mathrm{pm} / \sqrt{\mathrm{Hz}}$ and non-linearities below $10 \mathrm{pm} \mathrm{[3][9].} \mathrm{The} \mathrm{non-linearity} \mathrm{of} \mathrm{a} \mathrm{similar} \mathrm{het-}$ erodyne interferometer was previously determined to $50-$ $100 \mathrm{pm}$ using a gas cell [10]. The setup is placed in a vacuum chamber and operated at high vacuum conditions to reduce path length noise caused by air density fluctuations. During measurement the vacuum pump is deactivated and the valve is closed to reduce noise caused by vibrations.

Translation noise levels of the test setup, as shown in fig. 2, are below $10 \mathrm{pm} / \sqrt{\mathrm{Hz}}$ in the measurement band $(10 \mathrm{mHz} .6 \mathrm{~Hz})$ for the differential beams (x1-x2). The noise level of the asymmetric interferometer, required for path length corrections, is below $1 \mathrm{~nm} / \sqrt{\mathrm{Hz}}$.

Lower frequency drifts, as caused by pressure trends and temperature drifts, are removed in post processing. The measurement is acquired at $100 \mathrm{~Hz}$ and low pass filtered to approximately $6 \mathrm{~Hz}$ for suppressing environmental mechanical noise sources (mechanical/acoustical) at $14 \mathrm{~Hz}$.

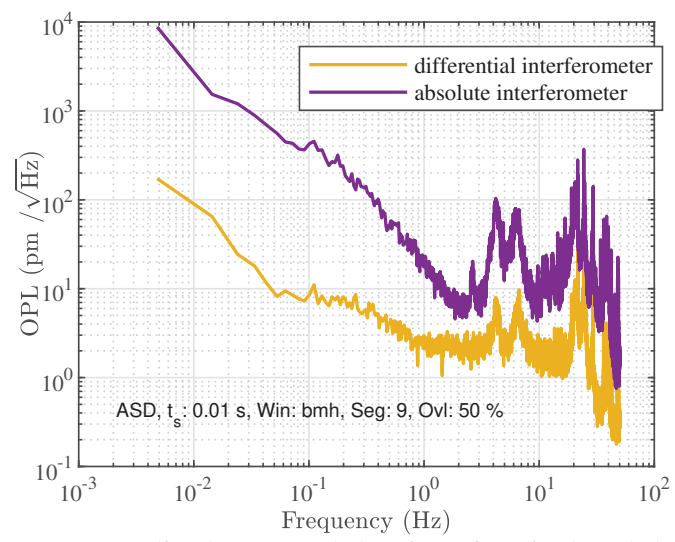

Figure 2: Amplitude spectral density of optical path length measured at steady-state.

Optical Setup The central optical setup probes the sphere surface at 3 positions as shown in figure 3. Two beams $(\mathrm{x} 1, \mathrm{x} 2)$ measure the optical path length to the sphere surface through a lens in a symmetrical differential configuration. The measurement beam uses polarisation routing (quarter wave plate) and a collimating lens. The lens is a 1 " best form lens with a focal length of $\mathrm{f}=150 \mathrm{~mm}$. The resulting azimuthal distance is $56 \mathrm{mrad}$ on the surface. After reflection on the surface and a second lens pass, each measurement beam is combined with a beam of the second frequency at mixing beam splitter and sensed by the final optical readouts Quadrant Photodiode (QPD) $(1.1,1.2)$. The two parallel measurement beams are separated by $\approx 10 \mathrm{~mm}$ and pass optical components collectively and are parallel within less than $100 \mu \mathrm{rad}$. The lens for the beams $(\mathrm{x} 1, \mathrm{x} 2)$ is positioned so that focal point and geometrical center of the sphere coincide. This should guarantee a match of wave-front curvature of the measurement beams to the surface of the sphere, however the wave-front is slightly mismatched since a single lens can not suit both functions for both beams perfectly leading to a slight reduction in heterodyne efficiency.

A third beam (y) from a second interferometer is perpendicular to the differential beams. A forth beam (y-ref) is used for reference and is length matched to the (y) beam. Orthogonality of the beams ( $x$ to $y$ ) is aligned with a penta prism. The (y) beam lens is adjusted to optimise the wave-front curvature matching.

The beam spot diameter on the surface of $140 \mu \mathrm{m}$ is calculated from an OptoCAD model.

\section{CIRCLE PROFILOMETRY}

Measurement Each greater circle is recorded at a constant velocity in azimuth direction. The measurement is performed over 3 revolutions at 5 degree per second. The acceleration/deceleration of each movement is discarded and only plateaus of constant velocity are evaluated. The azimuth position is recorded with a precision incremental encoder with an accuracy better than one arcsecond. The binned measured values are used to calculate a mean and standard deviation for each bin to evaluate the accuracy of the difference measurement itself. The measurement results of one ring measurement is shown exemplary in figure 4 . The average per pixel repeatability is better than 1 nanometer.

Circle profilometry corrections The measurement representing the differential signal as the difference between beam length $\mathrm{x} 1$ and $\mathrm{x} 2$ is geometrically insensitive to common path errors in the measurement direction. However a lateral movement will introduce different path length changes in each of the beams. The sources of movement are primarily the residual eccentricity of the sphere, stage movement errors (wobble) and deformation of the measurement platform. Geometrical contributions from sphere position are dominated by movement in the direction of $y$ in the order of approximately $3 \mu \mathrm{m}$, see figure 7 . The theoretical added path length for an ideal sphere can be described with a linearised correction factor $k_{y}=\sin \left(\varphi_{x 1, x 2}\right)$, where $\varphi_{x 1, x 2}$ is the azimuthal angle between beams $\mathrm{x} 1$ and $\mathrm{x} 2$. The corrected measurement based on the optical path lengths $d_{x 1|x 2| y}$ 


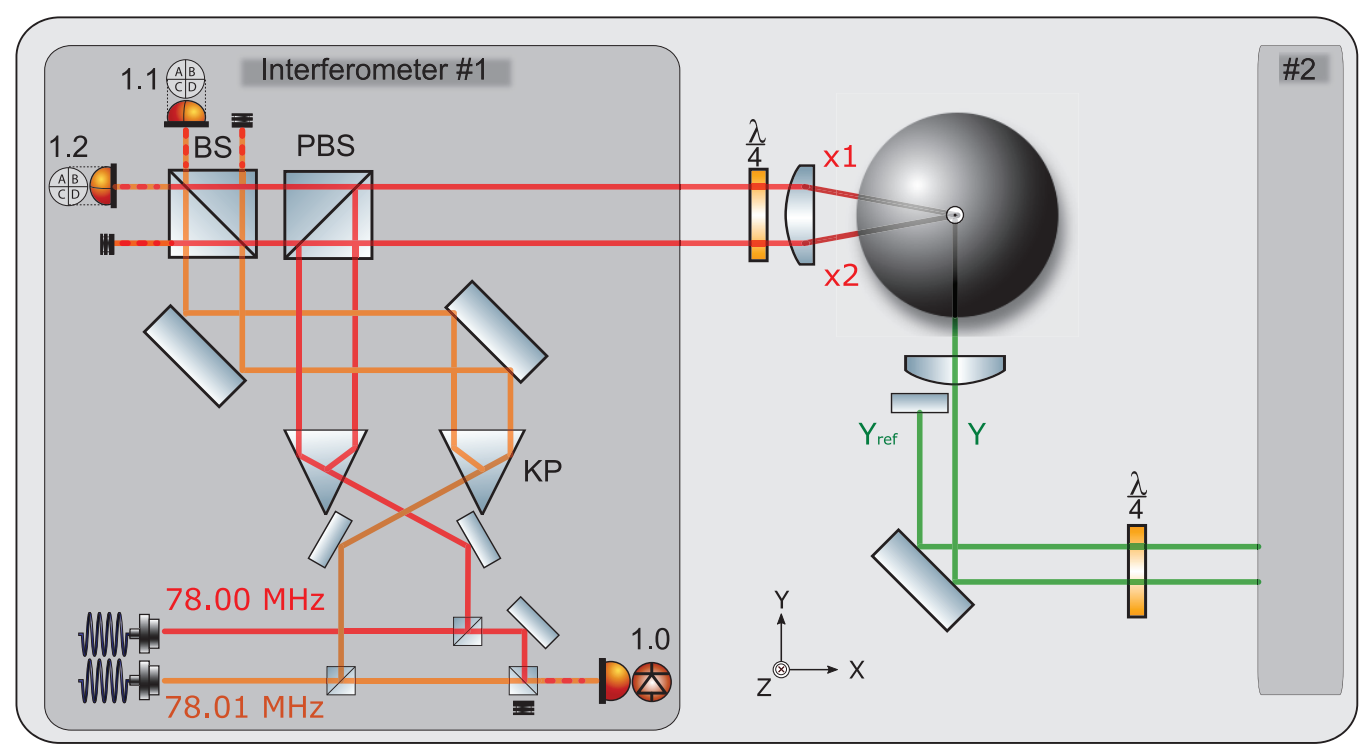

Figure 3: Schematic of the optical setup. The Beams (x1-x2) are in symmetrical differential configuration.
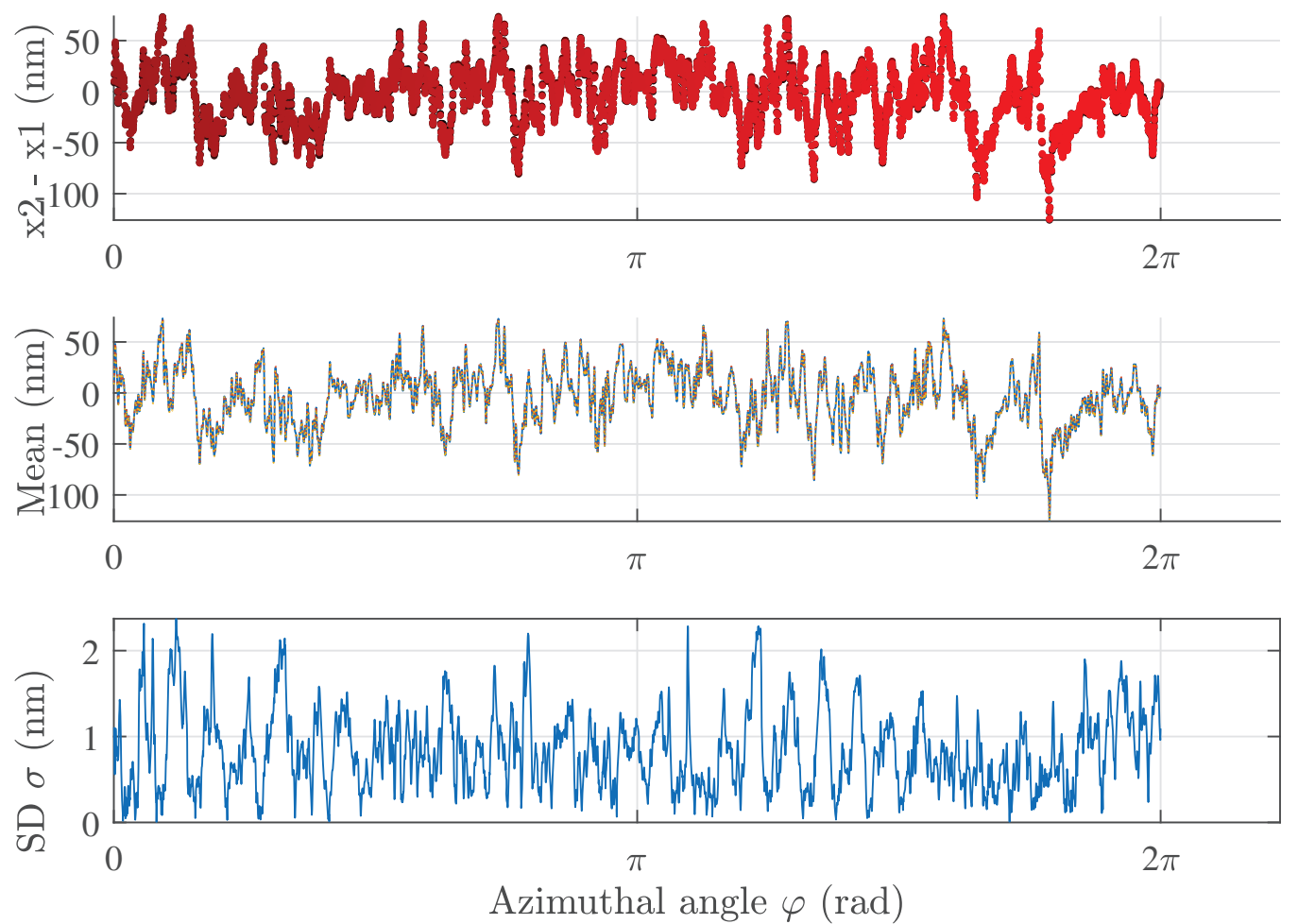

Figure 4: Performance of differential measurement for one exemplary circle profile. (Middle: Mean over 3 revolutions, Bottom: Standard deviation). 


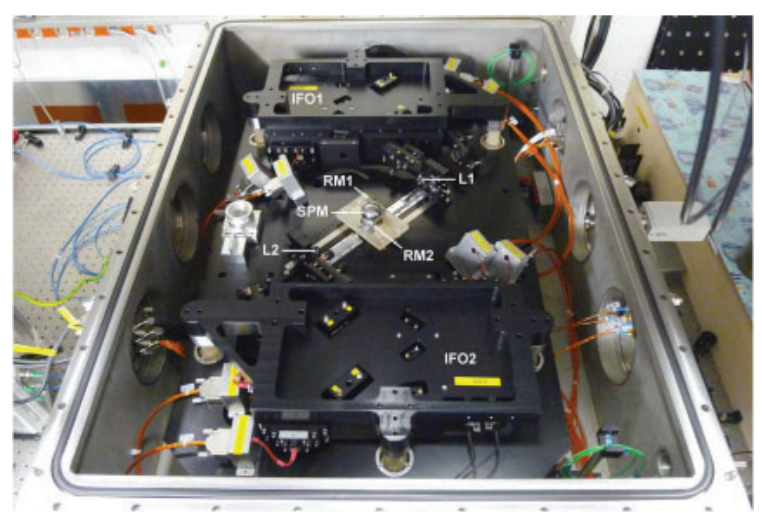

Figure 5: Measurement setup in the vacuum chamber showing the two interferometer blocks and the central table.

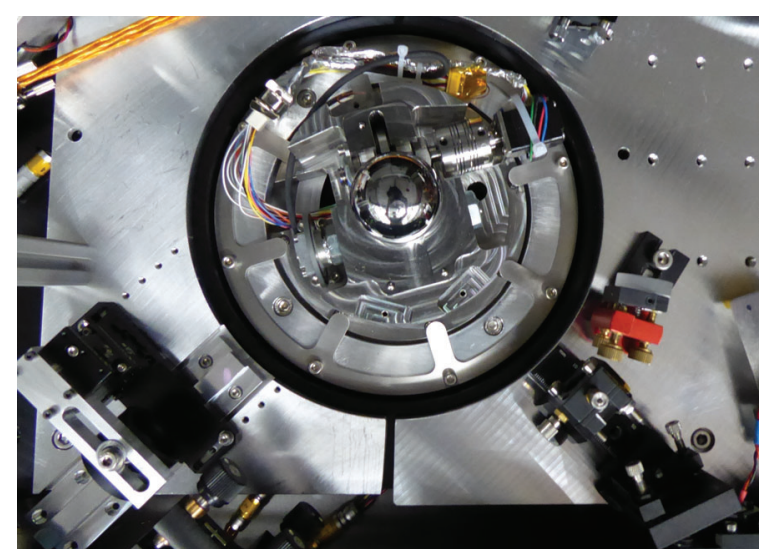

Figure 6: Optical setup with two mechanisms (elevation, azimuth) to rotate the sphere under test.
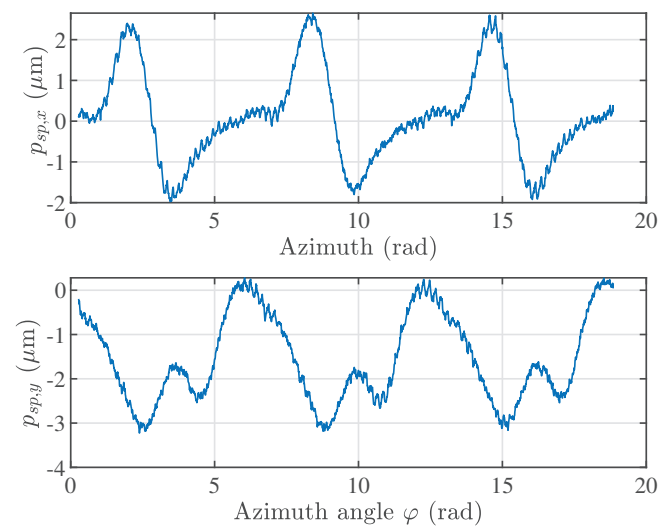

Figure 7: Measured position of the sphere. is given in eq. (1), where the sphere position $p_{s p, y}=d_{y}$.

$$
d_{\text {diff }}=d_{x 1}-d_{x 2}-2 \cdot k_{y} \cdot d_{y}
$$

The setup allows precise determination of the sphere relative position as shown in figure 7 . The error movement is a combination of sphere eccentricity effects, which cause repeating errors $\frac{1}{r e v}$ and $\frac{2}{r e v}$, and movement errors of the rotation stage. The total movement is approximately below $\pm 2 \mu \mathrm{m}$.

$$
\begin{aligned}
& p_{s p, y}=d_{y} \\
& p_{s p, x}=\frac{d_{x 1}+d_{x 2}}{2}
\end{aligned}
$$

A second contributing factor is the tilt caused by the different deflection angles on the sphere, this leads to additional tilt-to-length effects. The measurement is dominated by a linear correlation with the displacement of the sphere $p_{s p, y}$ and and the spheres radius $r_{s p}$. As a simple approximation the surface normal angle $\Phi$ depends on the curvature of the sphere, causing a returning beam angle of $2 \Phi$.

$$
\sin (\Phi)=\frac{p_{s p, y}}{r_{s p}}
$$

The impact on the path length has been simulated with a geometrical ray tracer [11]. A parameter study shows that in addition to geometrical contributions (described by the first intersection with the surface) due to the spherical shape, additional terms in the lateral direction depending on the position of the sphere relative to its ideal initial position exist as expected. The optical path length is not fully described by the previous geometrical aberrations: simulations of total geometrical and optical path lengths return a hyperbolic paraboloid for the differential measurement $d_{x 1}-d_{x 2}$ based on the sphere position $p_{s p, x}$ and $p_{s p, y}$. Additional minor terms exist in the longitudinal direction caused by the beam shape, but are not considered here and only the central ray is traced and evaluated. Higher order correction, or model-based correction, can not be implemented, since the position of the sphere $p_{s p}$ is only measured relatively. The accuracy of sphere position measurement is initially limited by the previously described surface and tilt-to-length effects, lending itself to be improved by iterative methods, after a first surface map has been established. The correction used is therefore a 2D linear fit of the optical path length as shown in figure 9 and evaluated individually for each circle. The resulting offset to length impact in the differential measurement amounts to $50 \mathrm{~nm} / \mu \mathrm{m}$ path length per sphere offset caused by sphere geometry by movement in horizontal plane. The optical impact attributes to approximately $1 \mathrm{~nm} / \mu \mathrm{m}$.

A linear trend in the non-equal length interferometer path length due to increase in vacuum pressure caused by the vacuum leak rate $(\approx 1 \mathrm{e}-5 \mathrm{mBar} / \mathrm{s})$ is present. This pressure increase is caused by the disabling the vacuum pumps during 
(a) $\mathbf{x 1}$

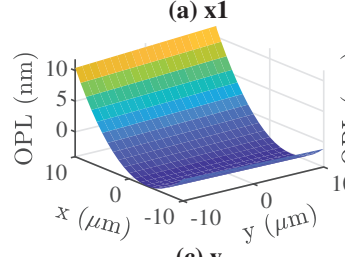

(c) $\mathbf{y}$

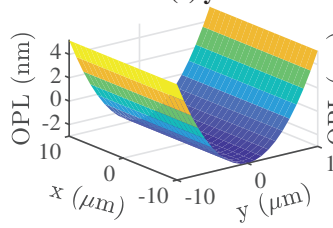

(b) $\mathrm{x} 2$

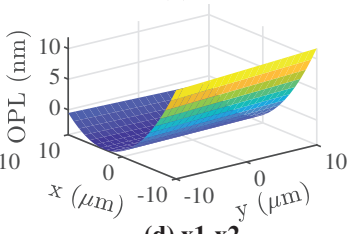

(d) $\mathbf{x 1 - x 2}$

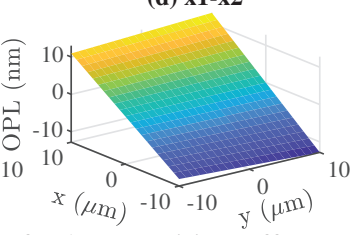

Figure 8: Simulated impacts of sphere position offset on optical path length readout for each beam $(\mathrm{a}-\mathrm{c})$ and differential measurement (d) x1-x2.

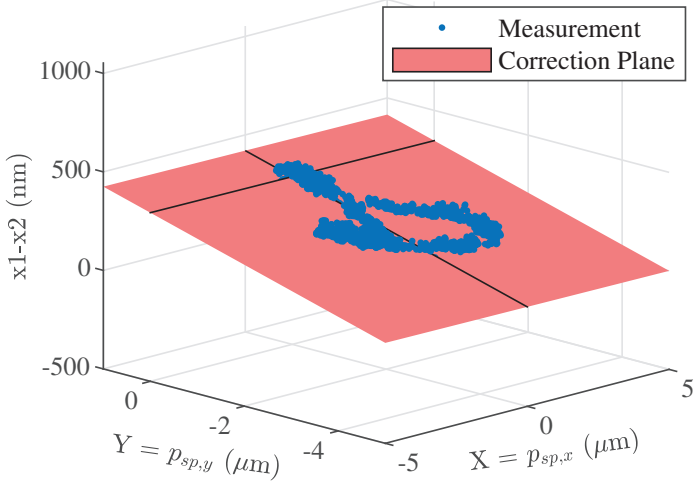

Figure 9: Correction of a circle profile measurement by a fitted 2D (X-Y) linear correction.

the time of measurement. In particular, the absolute path length measurement of the $d_{y}$ measurement arm is susceptible to pressure variations due to its unbalanced length. The corrected and binned values are detrended to compensate for any linear drift based on the assumption that at each location the measurement should yield same values over subsequent rotations. The average trend $d_{\text {trend }}$ per azimuth revolution is determined and then subtracted from the measurement. The trend is determined by comparing the value $d_{\text {diff }}$ at current position $\varphi(n)$ for the current pixel $n$, and subtracted by the value one revolution later. The average of all values is the correction used for the trend over one revolution.

$$
d_{\text {trend }}=\frac{1}{n} \sum_{n=0}^{\text {end }} d_{\text {diff }}(\varphi(n)+2 \cdot \pi)-d_{\text {diff }}(\varphi(n))
$$

Binning and consolidation Since the post-processing requires equidistant data the data is binned into a grid of pixels using linear interpolation over the azimuthal position $\varphi$. The number of pixels along the azimuth axis per full revolution $n_{p x}=4091$ is chosen to match the low pass filter frequency times the duration per revolution. The continuous grid is then mapped onto a single full revolution $(0 . .2 \pi)$ by modulo operation. During consolidation, the mean and deviation for each pixel is calculated. Performance of the consolidated differential measurement is shown in fig. 4 .

Reconstruction Two methods have been evaluated to reconstruct the surface profile from the difference measurement. One method performs a discrete reconstruction. A second method is based on Fourier transformation. Both methods require knowledge of the distance of the two beams on the surface. The $\varphi_{x 1, x 2}$ azimuthal angle between beams $\mathrm{x} 1$ and $\mathrm{x} 2$ has been determined by auto-correlating the measurements $(\mathrm{x} 1, \mathrm{x} 2)$ and simple geometrical considerations to $\varphi_{x 1, x 2} \approx 56 \mathrm{mrad}$.

Fourier profile reconstruction With the measurement consolidated into an equidistant grid over one revolution $d_{\text {diff }}(n)$, the measurement of the surface $s(\varphi)$ at two locations is described in (6) as a convolution. Hence the surface reconstruction can be described in the Fourier-domain as in (8). An machine epsilon $\varepsilon$ is added for numeric stability to avoid division by zero.

$$
\begin{aligned}
d & =d_{x 1}-d_{x 2}=s(\varphi)-s\left(\varphi+\varphi_{x 1, x 2}\right) \\
d(\varphi) \stackrel{\mathfrak{F}}{\longrightarrow} \widetilde{D} & =\widetilde{S}+\widetilde{S} \cdot e^{-j \cdot \omega \varphi_{x 1, x 2}} \\
\widetilde{S} & =\frac{\widetilde{D}}{1+\varepsilon-e^{-j \cdot \omega \varphi_{x 1, x 2}}} \stackrel{\mathfrak{F}^{-1}}{\longrightarrow} s(\varphi)
\end{aligned}
$$

Discrete profile reconstruction The basic principle of reconstruction is to iterate through the surface and constrain a distant surface position based on the current position and measured beam difference as in (6). This directional (forward) construction is initially performed over a single rotation, where the initial surface estimate is empty, $s(\varphi)=0$, and is described by the recursive algorithm:

$$
s\left(\varphi+\varphi_{x 1, x 2}\right)=s(\varphi)-d(\varphi)
$$

After this reconstruction adjacent spots of the reconstructed surface are not related to each other. This error causes a repetitive pattern in the first derivative $s^{\prime}=\mathrm{d} s / \mathrm{d} \varphi$ along the azimuthal rotation. It is caused by the wrong initial estimate (flat), and repeats in blocks with a length that corresponds to the distance of the two spots on the surface. The error pattern is recovered and used for smoothing, by averaging the derivative at position with multiples of $\varphi_{x 1, x 2}$.

$$
s_{0}^{\prime}(\varphi)=\frac{1}{b} \sum_{n=1}^{b}\left[d^{\prime}(\varphi)+\ldots+d^{\prime}(\varphi+n \cdot)\right]
$$




$$
\text { for } \varphi \in\left[0, \varphi_{x 1, x 2}\right] \text {. }
$$

Where $b=n_{p x} / n_{d}$ is the maximum integer number of repetitions that the beam distance $n_{d}$ can fit in the measurement data total pixel count $n_{p x}$. The new initial surface $s_{0}$ is the discrete integration of the pattern found in the derivative $s^{\prime}$. Only the initial values (with a total length of the beam distance) must be replaced, as later values are overwritten by the reconstruction.

$$
s_{0}(\varphi)=\int_{0}^{\varphi_{x 1, x 2}} \mathrm{~s}_{0}^{\prime}(\varphi) \mathrm{d} \varphi
$$

When a new forward integration is performed (eq. 9) but with new initial conditions $s(\varphi)=s_{0}$, the surface is finally recovered.

Comparison The performance of the reconstruction of both approaches (and for different assumed distances $\left.\left(\varphi_{x 1, x 2}\right)\right)$ is verified by generating a virtual measure from each reconstructed surface (fig. 10). The resulting standard deviation for both reconstruction methods is approximately $\sigma=0.38 \mathrm{~nm}$ for discrete reconstruction and $\sigma=9 \mathrm{~nm}$ for the Fourier reconstruction method. While in general the Fourier approach has the advantage of reconstructing a continuous repeating signal as is present with a circle the discrete method has proven to be more robust. The current discrete implementation deviates slightly in particular at the upper and lower limits $(0 / 2 \pi)$ as no special handling for the wrapping region is implemented. If the number of pixel bins used for evaluation is a multiple of the discrete beam distance, the measurement can be segregated into independent sets of measurement. To ensure correlation between all data the segment count can be chosen as a prime number. In figure 12 an exemplary set of reconstructed profiles, using the Fourier method, is shown.

\subsection{Sphere Surface}

After reconstructing all great circle profiles over several colatitudes $\theta$, a map can be generated. This recorded map of great circles shows a pattern that is repeated over both half domes as expected, and gives an indication of $\varphi_{e l}$, the azimuthal position of the elevation rotation axis. Fig. (13) shows a preliminary data set. The data is also used to calibrate the elevation readout encoder via the periodicity of the data.

The elevation axis is aligned perpendicular to the azimuth zero position to avoid artefacts generated by reconstruction. The exact angle can be extracted from the data assuming that all profiles intersect at the two poles, hence the reconstruction is constant over all great circle profiles at these points.

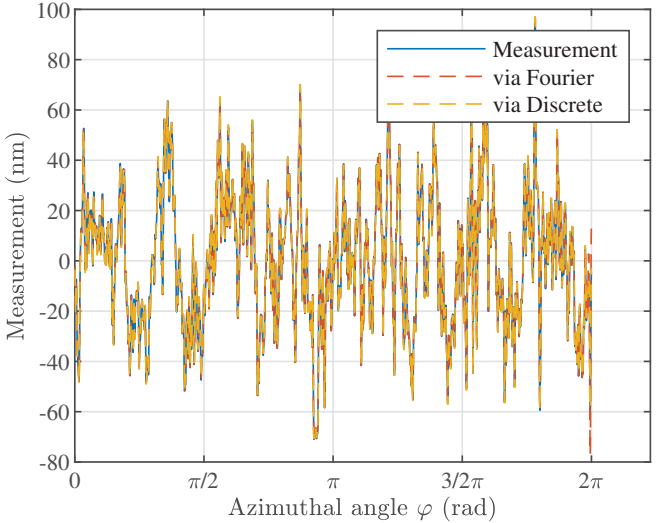

Figure 10: Original differential measurement and virtual measurements from reconstructed surface using two different methods for comparison.

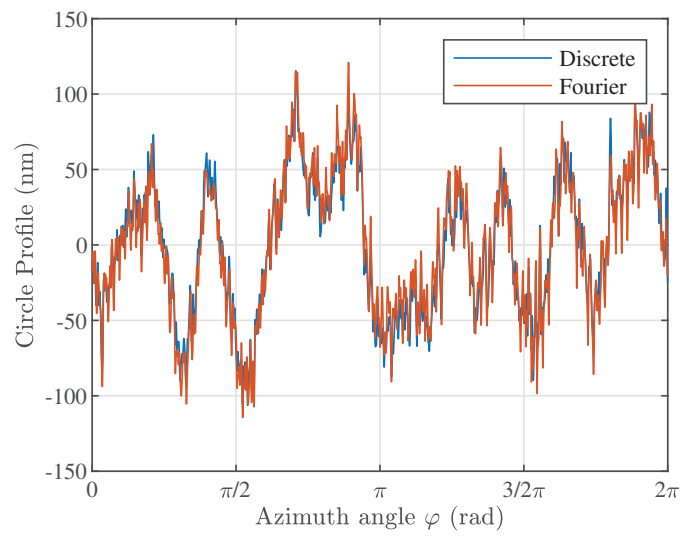

Figure 11: Reconstructed sphere surface along a circle of latitude.

The matrix $\mathbf{S}_{\rho, \varphi}$ denotes 2-dimensional collection of measurements along azimuth and elevation. The elevation axis is indicated where the deviation across all elevation position $\rho$ for one azimuth $\varphi$ position becomes minimal and is found with:

$$
\begin{gathered}
\varphi_{e l}=\underset{\varphi}{\operatorname{minimize}} \sigma\left\{\mathbf{S}_{\rho, \varphi}-\mathbf{S}_{\rho,[(\varphi-\pi) \bmod 2 \pi]}\right\}, \\
\text { with } \sigma\{x\} \text { the standard deviation of (x). }
\end{gathered}
$$

The measurements $\mathbf{S}$ are then circularly shifted along the azimuthal direction $\hat{\varphi}$ to align the second rotation axis at zero.

$$
\hat{\varphi}=\left(\varphi-\varphi_{e l}\right) \bmod 2 \pi
$$

Next, all profiles are adjusted so that $\hat{\mathbf{S}}_{\rho, 0}=0$, hence the initial pixel values for all profiles are aligned.

$$
\hat{\mathbf{S}}_{\rho, \hat{\varphi}}=\mathbf{S}_{\rho, \hat{\varphi}}-S_{\rho, \hat{\varphi}=0}
$$




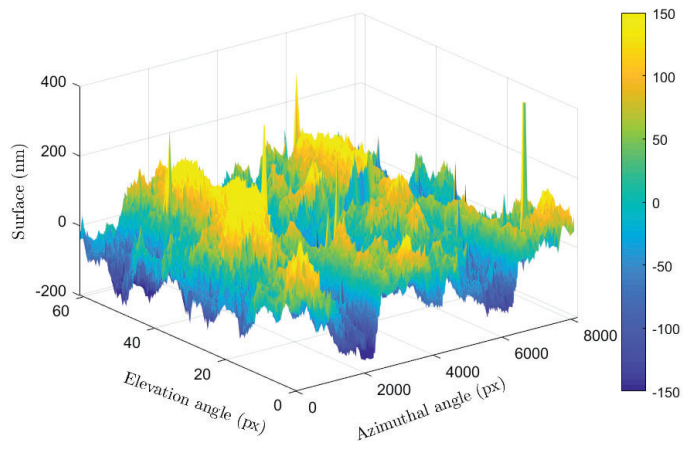

Figure 12: Reconstructed profiles over consecutive elevation measurements.

Finally each great circle pixel is translated into spherical coordinates $(\mathrm{r}, \theta, \varphi)$. Although the sphere radius is $r_{s p}=$ $20 \mathrm{~mm}$, we select smaller values of polar coordinate radius for exaggerating the representation (here $r_{s p}=2 \mu \mathrm{m}$ ). It is assumed that each great circle measured is an equator ( $\theta=\pi / 2$ ) and $\varphi$ corresponds to the measured elevation. Then each circle profile is rotated along the rotation axis $\vec{r}(\theta, \varphi)=[0, \pi / 2]$ by Rodrigues rotation.

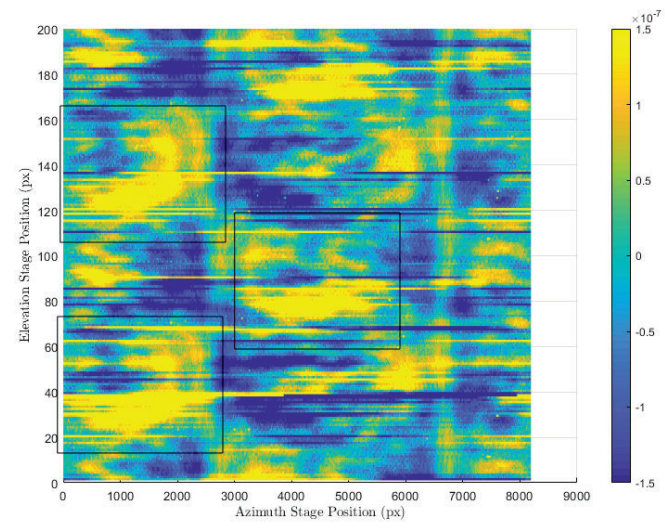

Figure 13: First preliminary surface profile data in the mechanism reference frame (azimuth, elevation).

Preliminary results and outlook We have presented a method for surface map acquisition by combination of greater circles at different elevations, which have been recorded and reconstructed using a differential approach with two beams. While single great circles show an excellent data quality (see fig. 4 , the process of reconstructing a full spherical surface needs further refinement, as apparently the data quality is yet insufficient for a proper reconstruction. Reconstruction artefacts and offsets when stitching together circles of different elevations, mostly caused

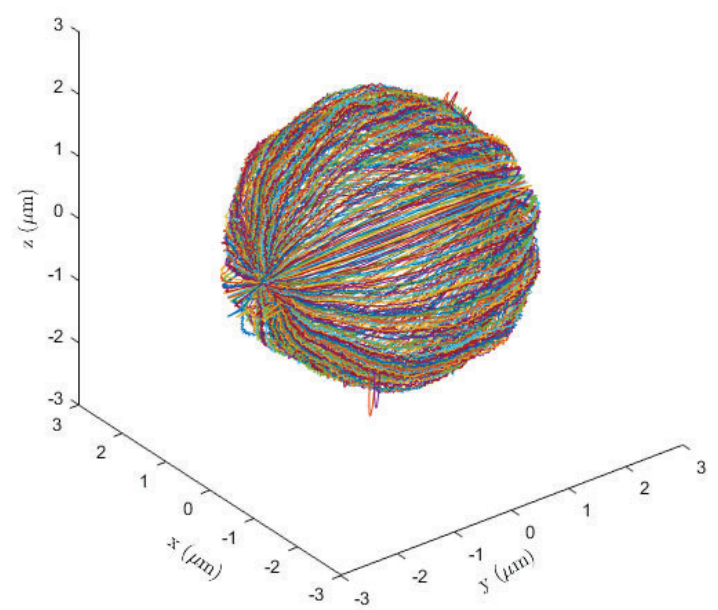

Figure 14: Visualisation of measured surface showing $\mathrm{n}=200$ circle profiles at different elevations. The sphere base diameter is reduced for exaggerated representation.

by offset-to-length effects that are not yet fully suppressed. Methods for reducing susceptibility to tilt-to-length could be to optimise the beam focusing using a second lens for each beam, and by reducing the total distance between the sphere and the recombining beam splitter. When using only one lens for geometrical centering and Gaussian wave-front curvature matching, two independent sets of constraint occur. Geometrically the focal point of the focusing lens must match the center of the sphere for incoming parallel beams. However matching the Gaussian wave-front would place the lens slightly further away from the sphere surface. Possible solutions for the given setup are separate lenses for each beam (loosing the common path symmetry between both beams), or alternatively to make the two beams slightly divergent instead of parallel using only a single lens. The reconstruction algorithms basically rely on integration, and thus are susceptible to adding up small systematic errors. Also minor errors in determining the beam offset or the measurement affect the results noticeably. Thus, in a next step, more robust algorithms and an improved beam layout will be investigated, in order to fully exploit the commonmode rejection demonstrated for the differential profilometry scheme presented here. 


\section{References}

[1] D. Gerardi et al. "Advanced drag-free concepts for future space-based interferometers: acceleration noise performance". In: The Review of scientific instruments 85.1 (2014), p. 011301. DOI: $10.1063 / 1$. 4862199.

[2] Y. Azuma et al. "Improved measurement results for the Avogadro constant using a 28 Si-enriched crystal”. In: Metrologia 52.2 (2015), pp. 360-375. ISSN: 0026-1394. DOI: $10.1088 / 0026-1394 / 52 / 2 / 360$.

[3] Marco Pisani et al. "Comparison of the performance of the next generation of optical interferometers". In: Metrologia 49.4 (2012), pp. 455-467. ISSN: 00261394. DOI: $10.1088 / 0026-1394 / 49 / 4 / 455$.

[4] John W. Conklin et al. "Determination of Spherical Test Mass Kinematics with Modular Gravitational Reference Sensor". In: Journal of Guidance, Control, and Dynamics 31.6 (2008), pp. 1700-1707. ISSN: 0731-5090. DOI: 10.2514/1.34230.

[5] R. Schachtschneider et al. "Interlaboratory comparison measurements of aspheres". In: Measurement Science and Technology 29.5 (2018), p. 055010. ISSN: 0957-0233. DOI: 10 . $1088 / 1361-6501 /$ aaae 96.

[6] Harald Kögel et al. "Interferometric surface mapping of a spherical proof mass for ultra precise inertial reference sensors". In: Applied optics 55.34 (2016), pp. 9830-9838. DOI: 10.1364/A0.55.009830.

[7] Torsten Mai, Rainer Tutsch, and Richard Kowarschik. "Aufbau und Simulation des Kugelinterferometers II der PTB". Dissertation. Technische Universität Braunschweig, 2016.

[8] C. Brun et al. "Picometer-scale surface roughness measurements inside hollow glass fibres". In: Optics express 22.24 (2014), pp. 29554-29567. DOI: 10 . 1364/OE. 22.029554.

[9] Dennis Weise et al. "A high sensitivity heterodyne interferometer as a possible optical readout for the LISA gravitational reference sensor and its application to technology verification". In: International Conference on Space Optics - ICSO 2008. Ed. by Josiane Costeraste, Errico Armandillo, and Nikos Karafolas. SPIE, 2017, p. 74. ISBN: 9781510616219. DOI: $10.1117 / 12.2308257$.

[10] Thilo Schuldt. "An Optical Readout for the LISA Gravitational Reference Sensor". PhD thesis. 2009.

[11] Alexander Schultze and Yuri Petrov. OptoMetrika Open source ray tracer for MATLAB. 2021. DOI: 10. $13140 /$ RG . 2.2.10027 . 98081/1. URL: https : //github.com/alexschultze/Optometrika. 